\title{
MPC AND ICQ DATABASES
}

\author{
B. G. MARSDEN, D. W. E. GREEN AND G. V. WILLIAMS \\ Harvard-Smithsonian Center for Astrophysics, \\ 60 Garden Street, Cambridge, MA 02138, U.S.A. \\ E-mail marsden@cfa.harvard.edu
}

\begin{abstract}
Databases of astrometric and orbital data for minor planets and comets and of photometric data for comets are maintained in connection with the publication of the Minor Planet Circulars and the International Comet Quarterly. Aspects of these publications and the content and availability of these databases are discussed.
\end{abstract}

\section{Astrometric observations of minor planets}

Astrometric observations of minor planets received by the Minor Planet Center (MPC) are generally published in the monthly batches of Minor Planet Circulars. Most of the observations made during the twentieth century have been photographic, but CCD data are rapidly increasing in number. In addition to a designation for each object, each observation contains the UTC of observation (usually midexposure, although sometimes the beginning and/or end of a trail will be used), generally to 0.00001 day; the $\mathrm{FK}_{5} / \mathrm{J} 2000.0$ right ascension and declination, generally to 0.01 second of time and 0.1 second of arc, respectively; and a three-figure code that defines the location from which the observation was made. Most of the positions are topocentric, although some have been reduced to the geocenter. $\mathrm{Di}$ scovery observations of new objects are denoted with an asterisk, some observations include a magnitude (assumed Johnson $B$ unless otherwise stated), and there may also be an observational note (e.g., bad seeing, time uncertain, etc.) or a code number that identifies an observation as made in the course of a particular program at the observatory location.

In the printed Minor Planet Circulars the observations are listed by observatory code and sublisted by designation (unnumbered objects first) and date. This format permits the convenient incorporation of more details concerning the observing programs. The observations are also made available in machine-readable form in the "Extended Computer Service" (ECS) operated by the MPC and the Central Bureau for Astronomical Telegrams (CBAT). Subscribers log in to one of the MPC/CBAT computers and give an address to which the data should be e-mailed. Options produce the files of observations of numbered and unnumbered minor planets from the current batch of Circulars. It should be noted that the distinction between numbered and unnumbered is that existing before the batch of Circulars was prepared, and minor planets given new permanent numbers in the batch will still therefore be unnumbered. The observations are filed in a format that is somewhat different from what is used on the pages of the Circulars. The ECS eliminates the notes shown in the printed Circulars but adds one indicating whether the observation has simply been transformed to J2000.0, or whether it was obtained directly in the $\mathrm{FK}_{5} / \mathrm{J} 2000.0$ system using a CCD, micrometer or meridian 
circle (photography being the default). The ECS also allows one to access the files from the previous batch of Circulars.

The MPC also merges the monthly files of observations into its full system of $\mathrm{J} 2000.0$ observational files. It is at this point that observations of newly-numbered minor planets (each of which may involve several different provisional designations) are transferred from the files of observations of unnumbered to those of numbered minor planets. The files are also amended according to the initial pages of the Minor Planet Circulars, as observations are corrected, deleted or redesignated.

Soon after the MPC was established at the Cincinnati Observatory in 1947 it was decided that files should be maintained of all observations of minor planets back to the beginning of 1939 , although earlier data were available in some cases, notably the extensive series of photographic data extending back to the 1890s obtained at the Heidelberg Observatory. It was not practical to carry out much checking, and most of the observations, even current data, were simply added to the file as published or as provided by the observers (the only modification of significance being a possible transformation of positions to equinox 1950.0). Frequently the observations were only approximate.

On the MPC's transfer to the Smithsonian Astrophysical Observatory in 1978 it was decided that current data should be subjected to some checking before publication and filing, that approximate positions would not be accepted, and that there should be at least some record of each provisional designation (even if this did involve approximate positions) back to the introduction of the modern designation system in 1925. Effort was also expended on checking the observations in that portion of the file inherited from Cincinnati, and many observations that had been attributed to low-numbered minor planets were (with suitable documentation in the Minor Planet Circulars) corrected, redesignated, reidentified and-in some cases - simply deleted. More and more pre-1939 photographic observations have been added to the files, and during the past year or two attention has also been given to the incorporation of older micrometric observations, re-reductions being automatically performed using positions of the reference stars updated from the $A G K_{1}$ (for example) to the PPM catalogue. Some meridian observations, notably those obtained in recent years with the Carlsberg Automatic Meridian Circle on La Palma and published elsewhere, are incorporated in the MPC files without republication in the printed Minor Planet Circulars.

The MPC makes complete copies of its files of astrometric observations, which as of October 1993 number 752,896, available on magnetic tape from time to time, although it has not been practical to update these exportable files more frequently than once every two or three years. Some users are able to make their own updates using the computer files issued monthly in the ECS, but this is not particularly easy to do. The ECS does therefore include an additional feature that allows subscribers to obtain from the latest MPC files all the available observations of objects specifically of interest to them. 


\section{Astrometric observations of comets}

Much of the preceding section applies also to astrometric observations of comets. One difference is that publication in the Minor Planet Circulars is by object and date. The observations made at the various locations are combined, with an initial list mentioning each program concisely. If magnitudes are provided, they are specified as either "total" $\left(\mathrm{T}=m_{1}\right)$ or "nuclear" $\left(\mathrm{N}=m_{2}\right)$, without consideration of the waveband to which the observations refer. Although quite detailed information concerning a comet's physical appearance has sometimes been included in the Circulars until very recently, it has been decided that this information is more appropriately published in the International Comet Quarterly (ICQ), and the notes published in the Minor Planet Circulars, like those for minor planets, tend to relate more to the "mechanics" of the observations.

Discoveries of new comets and recoveries of returning periodic comets are first announced in the CBAT's IAU Circulars. When comets are discovered visually by amateur astronomers the initial positions are necessarily very rough, and these positions are not carried over to the Minor Planet Circulars and MPC files. The $I A U$ Circulars also include some accurate positions, generally up to the point that an initial orbit (usually a parabola) can be computed with some reliability, and these positions are repeated in the Minor Planet Circulars.

The MPC files of cometary observations are complete only back to 1964 , although many earlier observations are also included. The total number of observations is 46,046. The ECS allows the files of cometary observations to be accessed, and the data are included on the occasional magnetic tapes that are issued.

\section{Orbital elements of minor planets}

Numerous sets of orbital elements of minor planets appear in the monthly batches of Minor Planet Circulars. These sets can include redeterminations for alreadynumbered minor planets, determinations for minor planets that are being newly numbered, identifications and linkages of observations at different oppositions, and results from observations at a single opposition. Except for the last group, the orbits are generally least-squares Newtonian representations and allow for the perturbations by the eight planets Mercury-Neptune and Ceres-although the effects of Pluto, Pallas and Vesta, the Earth and Moon separately, and in some cases relativity, may also be considered. In order that users can judge better on their reliability, the results for newly numbered minor planets and new multiple-opposition identifications are shown in full, with the $(\mathrm{O}-\mathrm{C})$ residuals listed for all the available observations. In the single-opposition cases perturbations are generally ignored, unless there is a close approach to the Earth or the length of the observed arc is more than 75 days. In some cases the computations are contrained by holding the orbital eccentricity fixed at some particular value.

Perturbed orbital elements are given in the $\mathrm{FK}_{5} / \mathrm{J} 2000.0$ system for standard epochs of osculation such that the Julian date is divisible by 200 with remainder 0.5 day; the angular elements are given to a precision of $0.00001 \mathrm{deg}$, and there is general compatibility for the other elements. Unperturbed orbital elements take 
for the epoch an instant when the Julian date is divisible by 20 with remainder 0.5 day, the angular elements being given to a precision of $0.01 \mathrm{deg}$.

Orbital data for individual minor planets (numbered, multiple-opposition unnumbered and recent single-opposition) can be obtained from the MPC/CBAT standard Computer Service (CS); there are also procedures therein for computing an unperturbed ephemeris and for listing the positions of all the numbered minor planets in a particular area of the sky at some time. The ECS provides orbital data from the current and immediately-previous Minor Planet Circulars, much as it provides observations. The current files contain orbital elements for the 5,700 numbered minor planets (one of which, 719 Albert, is lost) and for 16,347 unnumbered minor planets (not all of which will be unique). Still other options show all the provisional designations involved in the new numberings or the new identifications and double designations involving unnumbered objects.

Low-precision orbital data and identification information for all the unnumbered

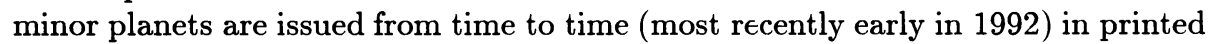
and diskette form as the Catalogue of Orbits of Unnumbered Minor Planets. The recent Catalogue of High-precision Orbits of Unnumbered Minor Planets (also in printed and diskette form. ) applies only to the cases where perturbations have been applied and to the standard epoch 1993 Aug. 1.0. This catalogue also provides some information about opposition dates and ephemerides; it is intended to be a companion to the annual Ephemerides of Minor Planets and STAMP software issued by the Institute of Theoretical Astronomy, St. Petersburg, for the numbered minor planets and will also be issued annually (with epochs updated to the last "200-day date" in each year) if there is sufficient demand.

The monthly schedule of the Minor Planet Circulars is not frequent enough for attending to newly-discovered Earth-approaching objects. These objects have traditionally therefore been covered on the IAU Circulars, which are themselves available in printed form and in the CS, delivery by e-mail also being possible. The increasing frequency of discoveries, and the hitherto largely unmet need for updating the data beyond an initial orbit determination, have overtaxed the $I A U$ Circulars. Accordingly, a series of Minor Planet Electronic Circulars (MPECs) has been inaugurated to provide these data more adequately. The MPECs are available in the CS and by e-mail but are not issued in printed form, on the grounds that all the important material in them (astrometric observations, final orbits) will appear in the next batch of Minor Planet Circulars.

\section{Orbital elements of comets}

Much of the preceding section applies also to orbital elements of comets. When perturbations are applied, which again usually requires observed arcs of 75 days or more, the osculation epoch is taken to be the "40-day Julian date" closest to perihelion passage. In unperturbed cases no epoch is specified, because of the convention of specifying the time of perihelion passage; likewise, the semimajor axis (and mean motion) is replaced by the perihelion distance. Predicted orbital elements are given around May of year $n$ for the comets expected at perihelion in the year $n+3$. Elements (and ephemerides) are also included in the annual ICQ 
Handbook if there is a reasonable chance that observations can be made in that year, but this Handbook is not available in computer form.

Cometary orbits in the CS include those of the long-period comets of the two or three preceding years and the reasonably predictable short-period comets, even when they are far from perihelion. Preliminary orbits are given with the astrometric observations in the IAU Circulars and thence in the CS. Cometary information of moderate urgency can also be given in the Minor Planet Electronic Circulars.

The complete Catalogue of Cometary Orbits has been issued eight times since 1972, and there seems to be interest in now having it updated annually. The 1993 edition, which contains 1,392 orbits for 855 individual comets (174 of them of short period), is available in printed form, by diskette and by e-mail. The diskette and the e-mail editions only contain the general catalogue and the "original" and "future" orbits of long-period comets in well-determined cases. The printed edition also supplies references and much other information, including the nongravitational parameters that should be taken into account when orbits are updated to other epochs.

\section{Photometric and other physical data on comets}

The ICQ was established in 1978 as a clearinghouse and archive for photometric observations of comets, filling a need for such an international center that had not previously existed. The establishment of the $I C Q$ archive has meant that many data previously published in the IAU Circulars and the Minor Planet Circulars are now usually published only in the $I C Q$, with minor exceptions. The $I C Q$ is published in January, April, July and October and contains general news and articles pertaining to the study of comets, although the central purpose of the $I C Q$ is the publication of tabulated data and descriptive textual information pertaining to the physical (photometric) observation of comets.

All of the tabulated data that are published in each issue of the $I C Q$ are sorted into the general $I C Q$ archive. A tabulated observation is an 80-character line that contains a coded identification for each comet, the date (given to 0.01 day in UT), a 5-character code for the observer, information concerning the instrument used, whether the observation was visual or non-visual (i.e., photographic, CCD, etc.), an estimate of the comet's total magnitude (along with coded information regarding the method used for making the estimate, the source from which comparison-star magnitudes were obtained for the estimate, and whether or not a correction was made for atmospheric extinction), estimates of the comet's coma diameter and tail length (with the position angle of the tail), and an estimate of the "degree of condensation". Of course, not all of the above information is provided with each observation, though observers are encouraged to report as many of these data as possible. For publication, it is required that observers contributing magnitude estimates provide the method employed, the source for comparisonstar magnitudes, and complete instrumentation details; data made prior to 1982 (or where the given comet is poorly observed) are exempt from this requirement. The requirement was established in an effort to standardize the way in which magnitude estimates are made, thereby improving the quality of the archive, and 
the scatter has been greatly reduced during the past decade as a result of this procedure.

As of October 1993, there were 59,869 observations in the archive, 94-percent of them containing some sort of magnitude information. "Negative" magnitude estimates (in which limiting magnitudes are estimated in situations where the comet was not detected) are present for 1,558 of these observations. Among the data containing magnitude estimates, there are 32,807 observations of 199 long-period comets and 23,656 of 125 short-period comets. The observations of long-period comets currently include comets from 1932 through 1993, while those of short-period comets begin with Halley's Comet in 1909. Halley's Comet has 6,779 observations with magnitude information in the $I C Q$ archive, leading all comets in this regard. Current efforts are aimed first at publishing all recent observations, and second at collecting older data from various groups worldwide (many of which have never been previously published). A third effort is being made at extracting older data from the literature, going back prior to 1900 , in which such published data enter the $I C Q$ archive directly without republication in the $I C Q$.

About a dozen "observation coordinators" have been set up throughout the world to collect observations within certain countries or regions, and these coordinators usually enter data in machine-readable form for relay to the $I C Q$ editor.

The data in the $I C Q$ archive are available in their entirety on 9-track magnetic tape to interested researchers.

\section{Availability of data}

Since the databases discussed in this paper have been prepared and maintained in connection with publications for which a subscription charge is necessary, the databases are themselves made available only by subscription. Those interested in subscribing to the data should contact one of the authors. 